\title{
Opportunities and challenges for energy harvesting sensor systems for harsh environments
}

\author{
Bahareh Zaghari \\ Department of Electronics and \\ Computer Science \\ University of Southampton \\ Southampton, SO171BJ, UK \\ bahareh.zaghari@soton.ac.uk
}

\author{
Alex Weddell \\ Department of Electronics and \\ Computer Science \\ University of Southampton \\ Southampton, SO171BJ, UK \\ asw@ecs.soton.ac.uk
}

\author{
Neil White \\ Department of Electronics and \\ Computer Science \\ University of Southampton \\ Southampton, SO171BJ, UK \\ nmw@ecs.soton.ac.uk
}

\begin{abstract}
Wireless sensing systems for harsh environments, especially at high temperatures, are of great interest to many industries. Wireless sensing systems consist of sensors, electronic interfaces and processors, energy harvesters, and wireless transmission modules. Realtime data collection from sensors, combined with data analytics, can improve safety and performance, and reduce operational and maintenance cost in harsh environments. Even though some sensors are available for harsh environments, it is still impossible to measure the real-time data wirelessly due to the lack of high temperature electronics and energy storage for the selected sensors. Typically, data is transferred with cables to cooler regions, where an external electronic box is set up. Due to complex wiring connections, reliability is poor, the sensor locations are restricted and the cost and weight of the sensing system is increased. In this paper, the limits of wireless sensing systems for high temperature applications are discussed and the opportunities for future research are outlined.
\end{abstract}

\section{KEYWORDS}

Wireless sensing systems, energy management, energy harvesting, harsh environment

\section{ACM Reference Format:}

Bahareh Zaghari, Alex Weddell, and Neil White. 2017. Opportunities and challenges for energy harvesting sensor systems for harsh environments. In Proceedings of ENSsys'17. ACM, New York, NY, USA, 3 pages. https://doi.org/10.1145/3142992.3143001

\section{INTRODUCTION}

Extreme environments are subject to a combination of extreme hot or cold temperature, high frequency, high power, high radiation and high electromagnetic interference (EMI), high mechanical stresses, and harsh chemical media. Typically these extremes cannot be efficiently reduced with additional subsystems, such as a shielding or cooling system at high temperature. Harsh environments exist in the aerospace, automotive, avionics, oil and gas, and steam power industries. Despite advances in technology, robust

Permission to make digital or hard copies of part or all of this work for personal or classroom use is granted without fee provided that copies are not made or distributed for profit or commercial advantage and that copies bear this notice and the full citation on the first page. Copyrights for third-party components of this work must be honored. For all other uses, contact the owner/author(s).

ENSsys'17, November 5, 2017, Delft, Netherlands

(C) 2017 Copyright held by the owner/author(s).

ACM ISBN 978-1-4503-5477-6/17/11.

https://doi.org/10.1145/3142992.3143001 and efficient wireless sensing systems are difficult to apply to harsh environments. In this study we address the challenges to design the circuit components in wireless systems for high temperature harsh environments, along with the current research and development in this area. In this paper, the wireless sensing module is described as a system, which comprises a sensor, integrated circuit, energy harvester, energy storage, and antenna. Design of sensors for high temperature environment depends on applications. The integrated circuit includes an amplifier, microprocessor and power management ICs. An analogue signal is measured from the sensor, amplified by the amplifier, converted into a digital signal by the analogue to digital converter on the microprocessor, filtered to address the quantization error, and output in digital format by the digital transmitter and antenna. Usually a sensing module for high temperature conditions contains a digital implementation on a wireless platform to achieve good quality data transmission. The energy harvester is implemented to provide in-situ energy for the integrated circuits and data transmission. Power management is necessary for electronics in harsh environments, since active electronics, integrated circuitry, and wireless communications all require power. Maximum Power Point Tracking (MPPT), which can be included in charge controllers are used for extracting maximum available power from energy harvester modules [17]. Power management ICs, that can provide MPPT are not yet available for harsh environments.The advances in high temperature electronics with considering the gap in the commercial market is explained in Sections 2.

\section{HIGH TEMPERATURE ELECTRONICS}

Conventional silicon based electronics fail to operate continuously above $80^{\circ} \mathrm{C}$. Instead of silicon researchers have proposed that cryogenic devices be used to increase compute throughput massively by using device architectures that could take advantage of the lack of thermal noise in circuits cooled below the temperature of liquid nitrogen. However, these solutions are expensive. Wide bandgap semiconductors such as diamond, group III-nitrides, and silicon carbide ( $\mathrm{SiC}$ ) offer great potential to fabricate active high temperature electronics $\left(>500^{\circ} \mathrm{C}\right)$. Furthermore, wide bandgap semiconductors can offer additional advantages in terms of high-power and high-frequency applications $[1,12]$. New development on $4 \mathrm{H}-\mathrm{SiC}$ junction field effect transistor (JFET) ICs with two levels of interconnect have started to consistently demonstrate longer $(>1000$ hours) operating times at $500^{\circ} \mathrm{C}$, which is a significant step towards jet engine design in the aerospace industry. Operational testing of $4 \mathrm{H}-\mathrm{SiC}$ JFET ICs at ambient temperatures up to $961^{\circ} \mathrm{C}$ have 
been reported [7]. Current packaging technologies are the limiting factors for the industrial applications of high temperature electronics. Plastic packages that are typically used are not suitable for applications above $150^{\circ} \mathrm{C}$. Ceramic packages for temperatures up to $500^{\circ} \mathrm{C}$ have been proposed [18], but are restricted to low current applications due to the resistivity of the refractory metals used as conductors [18]. In order to design packaging for harsh environments, the elastic behaviour and long term stability of the micromechanical structures at elevated temperatures must be addressed [3].

There are few commercial products available for designing high temperature data acquisition systems. For example, the H.E.A.T. Evaluation Module, a high temperature signal conditioning and processor evaluation platform designed by Texas Instruments and released in 2011, is designed to withstand operating temperatures up to $210^{\circ} \mathrm{C}$. The H.E.A.T. EVM PCB is made with polyimide material and includes high temperature rated passive components. Six analogue input channels are provided for temperature sensors, pressure sensors, and accelerometers. The limitation of this module is the high power consumption of its ARM7 Microcontroller. There are a few microcontrollers available for high temperature applications that also consume low power, such as OMAPL137BPTPH from Texas Instruments with current consumption $20 \mathrm{~mA}$ at $3 \mathrm{~V}$ operating at temperature $-55^{\circ} \mathrm{C}-175^{\circ} \mathrm{C}$, and PC $5674 \mathrm{~F}$ from TELEDYNE e $2 \mathrm{v}$ with current consumption $3 \mathrm{~mA}$ at $2 \mathrm{~V}$, and operating at temperature $-55^{\circ} \mathrm{C}-175^{\circ} \mathrm{C}$. There are few hermertically sealed high temperature semiconductors, though MOSFET and schottky diodes designed at Wolfspeed Fayetteville Arkansas campus (formerly APEI) operate at temperatures up to $225^{\circ} \mathrm{C}$.

\section{ENERGY HARVESTING SOLUTIONS}

Due to the limited lifetime and regular replacements of batteries, an energy harvester can be replaced as a power source. A thermoelectric generator, which converts heat energy to electrical energy is a popular choice of energy harvesting compares to vibration, solar, and RF energy harvesting in high temperature environments [8]. Thermoelectric generators operate on the principle that, when the connected junctions of two dissimilar materials have a temperature difference, an electrical current is generated. However in most extreme temperature applications, maintaining this temperature variation is challenging [8]. Typically, the best performance of the thermoelectric generators is reached when one side of the thermoelectric module is in contact with forced air convection [22]. An encapsulated thermoelectric module consists of a vacuum-tight stainless-steel container in which the BiTe thermoelectric module is encapsulated. This construction enables maximum performance and durability, because the thermal expansion mismatch between the hot and cold sides of the container can be achieved using a sliding sheet in the container. The thermoelectric module inside is always kept in a vacuum environment so significant oxidation cannot occur, and the pressure difference between the inside and outside of the container reduces thermal contact resistance inside the container. Another design that is commercially available is a skeleton module in which there is an electrically insulating base plate on one side and the other side is open. If the base plate is attached to the colder side, it is easier to relieve the thermal stress in the module [4]. The power density of the commercial thermoelectric generators is in the range of $10\left(\mathrm{~mW} / \mathrm{mm}^{2}\right)$ for a significant temperature gradient.

Vibrational magnetic power generators based on moving magnets or coils can provide power density normalised with respect to the source acceleration $\left(\mathrm{W} / \mathrm{cm}^{3} \cdot \mathrm{g}^{2}\right)$ near 0.2 for room temperature applications $[11,14]$. Electromagnetic devices can be manufactured for temperature around $500^{\circ} \mathrm{C}$ considering alternative materials for the coils and magnets, however, the cost, size, and the weight of the device might be increased [6].

\section{ENERGY STORAGE SOLUTIONS}

Recently several battery systems have been developed which operate at high temperature $300^{\circ} \mathrm{C}-400^{\circ} \mathrm{C}$ under specific conditions. Batteries based on $\mathrm{Li}$ and $\mathrm{Li}$-alloy anodes are one of the power sources of choice for harsh environment applications due to their ruggedness, reliability, and high-power capabilities. High temperature molten salt batteries have been developed for electric vehicle applications. Similar to thermal batteries, the molten salt batteries consist of a high temperature molten salt electrolyte such as $\mathrm{LiCl}-\mathrm{KCl}$, a $\mathrm{Li}-\mathrm{Al}$ alloy anode, and a FeS2 cathode [2]. The limitation of these batteries is related to their discharge rate and operating range which should be between the melting point of the salt at approximately $400^{\circ} \mathrm{C}$ and the decomposition temperature of the positive electrode material (approximately $600^{\circ} \mathrm{C}$ for $\mathrm{FeS} 2$ ).

High temperature ultracapacitors (supercapacitors), or electrical double layer capacitors are also a good option for energy storage for wireless sensing modules [15]. Ultracapacitors store energy through electrostatic forces, in comparison with batteries which store energy through chemical potential. The maximum operating temperature for Ultracapacitors available on the market are usually between $65^{\circ} \mathrm{C}$ and $75^{\circ} \mathrm{C}$, though FastCap has developed an energy storage device operating up to $150^{\circ} \mathrm{C}$, and are working on a ultra-high temperature solid-state ultracapacitor module with operating temperature at $350^{\circ} \mathrm{C}$ designed specifically for harsh environments, albeit with limited capacitance and voltage range.

\section{WIRELESS TRANSMISSION}

Wireless devices have been proposed for health monitoring systems for sensor networks on board aerospace vehicles [19] and aircraft engines [9]. Wireless communication of data between devices consumes power, motivating a low-power communication design. To transmit data wirelessly, communication technologies such as IEEE 802.15.4, low power WiFi, 6LoWPAN, Radio Frequency Identification (RFID), Near Field Communication (NFC), Sigfox, LoraWAN, and other proprietary protocols for wireless networks have been developed [13]. Data is received and transmitted with an RF transmitter, however, off-the-shelf radio chips that withstand high temperatures are not available. Passive wireless systems, Surface Acoustic Wave (SAW) resonators [10], mechanical resonator MEMS devices [5], and LC resonant devices [16] have been introduced in literature for near field transmission. The research study conducted by Jie Yang [20] is an example of a wireless sensing device for harsh environments. The pressure sensor, a piezoresistive MEMS device, operates at $450^{\circ} \mathrm{C}$. The wireless transmission $\mathrm{SiC}$ FM module was designed and developed for low power devices. This example is has 
been extended to use a set of SiC-based integrated wireless sensortransmitter suites for extreme temperature operation $\left(450^{\circ} \mathrm{C}\right)$ in Nuclear Thermal Propulsion (NTP) engines. These sensor suites allow for real-time monitoring [21]. Further work for this study is to combine the system with an energy harvester [21], though there is a large amount of work needs to be done to provide reliable wireless transmission for extreme environments.

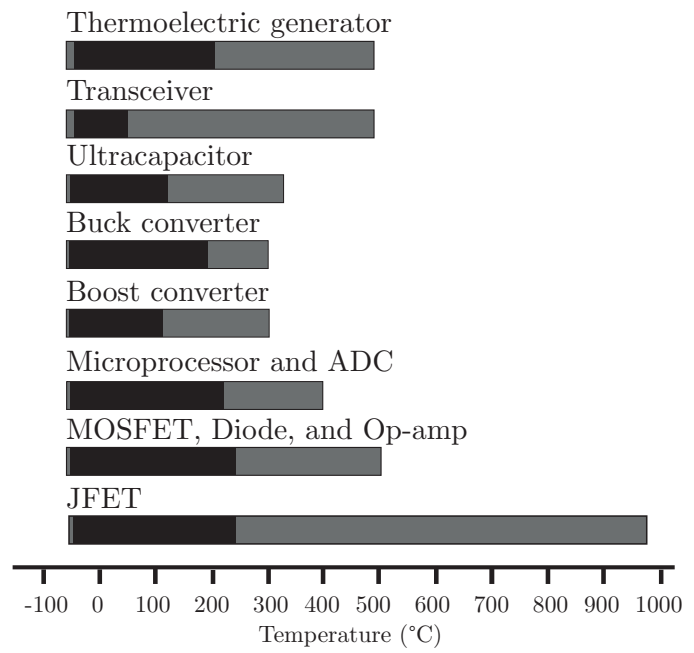

Figure 1: Operating temperature for commercial off-theshelf (black) and research (grey) devices.

\section{CONCLUSIONS}

Wireless sensing systems are required in many applications to efficiently obtain data and inform decisions, though the harsh environment of certain applications inhibits their use. Wireless sensing systems comprised of electronic components have a maximum operating temperature, which can be exceeded in particularly harsh environments, rendering traditional silicon electronics and plastic packaging unsuitable. Recently proposed technologies, including wide bandgap semiconductors and ceramic casings can increase the operating temperature of a given circuit considerably, but with other compromises on circuit design. Fig. 1 demonstrate the operating temperature for commercial off-the-shelf and research devices, which is necessary to design a wireless sensing system. These core mechanisms are supplemented by platforms designed by members of the industry. Energy harvesting and storage is of particular importance in harsh environments, as certain mechanisms cannot function under certain environments; for example, a magnetic power generator does not function well in an environment with a strong latent magnetic field. A review of vacuum thermoelectric generators operating in high temperature environments has been conducted in this paper. Recent developments in lithium-based batteries have resulted in some devices suited to high temperature operation. Some high temperature ultracapacitors are under development, though some compromise is required from the system designer between the operating temperature, the capacitance, and the voltage range. Given the compromises required when storing and harvesting energy in a harsh environment, an optimised power management system is recommended. The restriction on power impacts the wireless transmission and sensor devices that can be used in harsh environments, as care must be taken to ensure that both the operating temperature and the power requirements of these components is respected.

\section{REFERENCES}

[1] Wolfgang R. Fahrner, R Job, and Matthias R. Werner. 2001. Sensors and smart electronics in harsh environment applications. Microsystem technologies 7, 4 (2001), 138-144.

[2] Syozo Fujiwara, Minoru Inaba, and Akimasa Tasaka. 2010. New molten salt systems for high-temperature molten salt batteries: LiF-LiCl-LiBr-based quaternary systems. Fournal of Power Sources 195, 22 (2010), 7691-7700.

[3] Ping Hagler, Phillip Henson, and R Wayne Johnson. 2011. Packaging technology for electronic applications in harsh high-temperature environments. IEEE Transactions on Industrial Electronics 58, 7 (2011), 2673-2682.

[4] Mitsuru Kambe, Jinushi Takahiro, and Ishijima Zenzo. 2010. Encapsulated thermoelectric modules and compliant pads for advanced thermoelectric systems. Journal of electronic materials 39, 9 (2010), 1418-1421.

[5] Aaron J. Knobloch, Faisal R. Ahmad, Dan W. Sexton, and David W. Vernooy. 2013 Remote Driven and Read MEMS sensors for harsh Environments. Sensors 13, 10 (2013), 14175-14188.

[6] Jef Moser. 2003. Electromagnetic Devices for Operation in High Temperature Ambient 1000F. (2003). Retrieved August 25, 2017 from www.firstmarkaerospace.com/pdf/vhtmotors.pdf

[7] Philip G. Neudeck, David J. Spry, Liangyu Chen, Norman F. Prokop, and Michael J. Krasowski. 2017. Demonstration of 4H-SiC digital integrated circuits above 800 C. IEEE Electron Device Letters 38, 8 (2017), 1082-1085.

[8] David M. Rowe. 1995. CRC handbook of thermoelectrics. CRC press.

[9] Maximilian C. Scardelletti, Jennifer L. Jordan, George E. Ponchak, and Christian A. Zorman. 2015. Wireless capacitive pressure sensor with directional RF chip antenna for high temperature environments. In Wireless for Space and Extreme Environments (WiSEE), 2015 IEEE International Conference on. IEEE, 1-6.

[10] G Schimetta, F Dollinger, G Scholl, and R Weigel. 2000. Wireless pressure and temperature measurement using a SAW hybrid sensor. In IEEE Ultrasonics Symposium, 2000, Vol. 1. IEEE, 445-448.

[11] Genevieve A. Hart Scott D. Moss, Owen R. Payne and Chandarin Ung. 2015. Scaling and power density metrics of electromagnetic vibration energy harvesting devices. Smart Materials and Structures 24, 2 (2015), 023001.

[12] Debbie G. Senesky, Babak Jamshidi, Kan Bun Cheng, and Albert P. Pisano. 2009. Harsh environment silicon carbide sensors for health and performance monitoring of aerospace systems: A review. IEEE Sensors fournal 9, 11 (2009), 1472-1478.

[13] Pallavi Sethi and Smruti R. Sarangi. 2017. Internet of Things: Architectures, Protocols, and Applications. Fournal of Electrical and Computer Engineering 2017 (2017).

[14] Dirk Spreemann and Manol Yiannos. 2012. Electromagnetic vibration energy harvesting devices: Architectures, design, modeling and optimization. Vol. 35. Springer Science \& Business Media.

[15] Ranjith Vellacheri, Ahmed Al-Haddad, Huaping Zhao, Wenxin Wang, Chengliang Wang, and Yong Lei. 2014. High performance supercapacitor for efficient energy storage under extreme environmental temperatures. Nano Energy 8 (2014), 231-237.

[16] Ya Wang, Yi Jia, Qiushui Chen, and Yanyun Wang. 2008. A passive wireless temperature sensor for harsh environment applications. Sensors 8, 12 (2008), 7982-7995.

[17] Alex S. Weddell, Geoff V. Merrett, and Bashir M. Al-Hashimi. 2012. Photovoltaic sample-and-hold circuit enabling MPPT indoors for low-power systems. IEEE Transactions on Circuits and Systems I: Regular Papers 59, 6 (2012), 1196-1204.

[18] Matthias R. Werner and Wolfgang R. Fahrner. 2001. Review on materials, microsensors, systems and devices for high-temperature and harsh-environment applications. IEEE Transactions on Industrial Electronics 48, 2 (2001), 249-257.

[19] William C. Wilson and Peter D. Juarez. 2014. Emerging needs for pervasive passive wireless sensor networks on aerospace vehicles. Procedia Computer Science 37 (2014), 101-108.

[20] Jie Yang. 2013. A harsh environment wireless pressure sensing solution utilizing high temperature electronics. Sensors 13, 3 (2013), 2719-2734.

[21] Jie Yang. 2015. Extreme-Environment Silicon-Carbide (SiC) Wireless Sensor Suite, NASA technical reports server. (2015). Retrieved August 25, 2017 from https://ntrs.nasa.gov/search.jsp?R=20160005355

[22] Bahareh Zaghari, Alexander Weddell, Neil White, Imran Bashir, Terry J. Harvey, and Ling Wang. 2017. Integrated smart bearings for next generation aeroengines.: Part II: energy harvesting and wireless communication development. In First World Congress on Condition Monitoring - WCCM. 